\title{
The Role of Forensic Biomechanics/Medicine in Physical Child Abuse
}

\section{Hannon PR*}

Professor at Northern Arizona University, College of Engineering and Natural Sciences, Department of Biology, USA

"Corresponding author: Hannon PR, Professor at Northern Arizona University, College of Engineering and Natural Sciences, Dept. of Biology, USA, Tel: 480-816-0930/928-607-0425; Fax: 480-816-0931; E-mail: hannon@Hannonbiomechanics.com

Received date: Jan 01, 2014; Accepted date: Jan 06, 2014; Published date: Jan 12, 2014

Copyright: @2014 Hannon PR. This is an open-access article distributed under the terms of the Creative Commons Attribution License, which permits unrestricted use, distribution, and reproduction in any medium, provided the original author and source are credited.

\section{Editorial}

Many attorneys think of biomechanics in terms of personal injury/ wrongful death involving traffic accidents. This area is typically the majority of litigation support provided by many biomechanists and in our practice, it is still a significant minority of our cases. However, biomechanics along with forensic medicine (licensed pathologist) are able to provide a broader scope of expertise. One such area is physical assault (spouse/child abuse, other physical confrontations, etc.). We advocate a team approach in the case of physical child abuse which will ideally involve a pediatric physician, a forensic biomechanist, and a pathologist (MD or DO) with forensic certification. Furthermore, sometimes a pediatric radiologist will be able to provide valuable forensic input.

Recently, the medical community has had to reexamine the issues of physical child abuse especially in terms of skin pattern injuries, shaken infant-toddler syndrome (brain-brainstem injury) and bone fractures of the ribs and of long bones in children. A clarification of injury mechanisms from a biomechanics perspective and a medical pathology perspective is of utmost importance in distinguishing accidental injury v. non-accidental injury. In this regard, analysis should not stem simply from anecdotal examples where many variables were not and could not be fully addressed. Rather, when experimental data are available from infant/toddler cadaver testing data (e.g. skull fractures) or more typically from anthropometric child dummy test data, then the results have a solid scientific basis. In short, this is evidence based science and medicine.

In regard to head injury, statements made in the past by physicians that the force required to produce a single linear skull fracture or a mild/moderate depressed skull fracture in a toddler aged child was "equivalent to that child falling head first to the pavement below from a three story building" are without merit. Such statements are very misleading to the trier of fact. Instead, what is relevant involves evidence based force and acceleration data on shake and strike actions, shaking alone without a head strike, and free falls v. inverted pendulum falls onto a hard surface or down a stairway in terms of differentiating accidental injury from non-accidental injury. Furthermore, many times the fracture pattern of the skull will answer the question of how many head strikes occurred and at what skull location in addition to the range of forces required producing such fracture failure defects of the head/face.

Rib (costae) fractures as an indicator of child abuse has been questioned recently. It is important to understand for example how the location of rib fractures determines the probable mechanism of injury. Shaking and squeezing of the thorax of an infant can result in posterior fractures near the transverse processes of the spinal column. The mechanism of injury involves the transverse processes acting as a fulcrum as per a first class lever system and when these types of multiple rib fractures predominate, this becomes a strong indicator of non-accidental injury. Conversely, fractures which are along the lateral arc of the ribs are due to compression loads applied to the thorax resulting in increased bending of the ribs. Such fractures are the result of "uniform clamp" loading which results in compression and tension stresses along the respective internal and external rib surfaces. These lateral arc rib fractures do not rule out physical child abuse but accidental injury must also be seriously considered. Furthermore, in this latter case, multiple rib fractures may occur without skin pattern trauma due to a single broad based load which may have resulted simply from a single accidental compression load being applied (e.g. an older sibling accidentally sitting on an infant).

Long bone fractures during physical trauma may involve injury to the metaphysis (a bone growth plate interface) or to the bone long axis shaft. The bone fracture pattern may be an oblique, transverse or a spiral pattern along the shaft. Such fractures may be indicative of physical abuse or accidental injury depending upon the constellation of injuries. Ligamentous injuries may also result at joints when force moments or torques are applied by a perpetrator. Clearly, the overall pattern of injury must be carefully viewed from a medical and biomechanics perspective and competing or contributing etiology such as a multitude of disease processes or nutrition deficiencies must be carefully examined by the physician.

From the pathologist's perspective the following aspects of the case suggest abuse:

Presence of healing fractures and fractures with irregular calluses

\section{Fractures of different ages}

Presence of abuse pattern injuries on the surface of the skin

\section{Evidence of malnutrition (neglect)}

Failure to seek medical attention or significant delay in seeking medical attention

Finally, the coincident biomechanics analysis involves examining the detailed statements of defendants in terms of their story or stories. Human functional anatomy/biomechanics and fundamental Newtonian mechanics will either support or refute such stories. This involves the analysis of the kinematics (i.e. infanSt/child and the accused perpetrator) in proposed accident scenario(s). Sometimes a scenario story has merit and sometimes it does not. The biomechanics analysis of the kinematics is the hard science that plays a major role in the determination of physical abuse v. accidental injury. In this regard, police interviews of defendants should be detailed (without being manipulative), with follow-up probing and if possible video recorded 
Citation: Hannon PR (2014) The Role of Forensic Biomechanics/Medicine in Physical Child Abuse. Forensic Biomechanics 5: e101. doi: 10.4172/2090-2697.1000e101

Page 2 of 2

to permit the defendant to show as well as explain his/her story in terms of how the injury to the infant or child occurred.

Without question, it is very important to engage in a careful team approach analysis and make judgments that get it right for the child and the caregiver(s). As is obvious, an error in either judgment direction will result in great harm to the family. 